\title{
Optimization of the Crosslinking Process with Glutaraldehyde Vapor in PVA Based Electrospun Membranes to Wound Dressings Applications ${ }^{\dagger}$
}

\author{
Marta A. Teixeira, Joana C. Antunes, M. Teresa P. Amorim and Helena P. Felgueiras* \\ Centre for Textile Science and Technology (2C2T), Department of Textile Engineering, University of Minho, \\ Campus of Azurém, 4800-058 Guimarães, Portugal; martaalbertinateixeira@gmail.com(M.A.T.), \\ joana.antunes@2c2t.uminho.pt (J.C.A.); mtamorim@det.uminho.pt(M.T.P.A.) \\ * Correspondence:helena.felgueiras@2c2t.uminho.pt \\ + Presented at the 2nd International Online-Conference on Nanomaterials, 15-30 November 2020; Available \\ online: https://iocn2020.sciforum.net/.
}

Published: 15 November 2020

\begin{abstract}
Chronic wounds (CW) are characterized by delay or non-healing after 4 weeks of treatment. CW have become more prevalent, leading to a huge burden on healthcare and social systems, requiring specialized care. Wound dressings with specific properties capable of promoting regeneration and avoiding infections are highly needed. However, their efficacies are still limited due to the complexity of wound healing process and inadequate functions of wound dressings. To the effect, mats prepared at $10 w / v \%$ concentration of PVA/CA (100/0, 90/10 and 80/20 v/v\%) in glacial acetic acid and water at $75 / 25 v / v \%$ ratio were produced via electrospinning technique. The electrospinning setup consisted of a syringe and metal needle, an aluminum collecting plate, and a high voltage power supply. Conditions were optimized to obtain uniform, bead free mats, with a flexible structure and mechanical resilience. To overcome the instant solubilization of the PVA portion of the mat in aqueous media, a crosslinking process was employed. Crosslinking implies the formation of chemical bonds between different molecular chains to generate strong, stable and water insoluble 3D networks. For PVA, the crosslinking process can be accomplished via chemical or physical reactions, being the dialdehydes, diisocyanates, dicarboxylic, tricarboxylic, and boric acids the most frequent chemical agents applied. Even though there are various options, glutaraldehyde (GA) is by far the most common crosslinker used in PVA processing due to its efficiency, ease of access and processing, and low cost. Further, in comparison with other crosslinking agents, GA in its vapor form has demonstrate reduced or no cytotoxic effect. Therefore, GA vapor was the crosslinker chosen and various parameters such as the amount of GA, time, temperature, and drying methods were tested to guarantee its elimination. GA vapor at $25 \%$ in water for $7 \mathrm{~h}$ at $60^{\circ} \mathrm{C}$, using $6 \mathrm{~mL}$ of solution per $130 \times 120 \mathrm{~mm}^{2}$ of mat proved to be the most efficient option. To eliminate residual GA from the mats, storage conditions were defined in a controllable environment of $41 \% \mathrm{RH}$ and $19^{\circ} \mathrm{C}$ until usage.
\end{abstract}

Keywords: chronic wounds; electrospun biodegradable mats; crosslinking process; Glutaraldehyde vapor

\section{Introduction}

$\mathrm{CW}$ are the result of inadequate repair processes that are unable to restore the anatomic and functional integrity of the affected sites in an appropriate time. Despite differences in etiology at the molecular level between the various categories of $\mathrm{CW}$, they share certain common features, including excessive levels of pro-inflammatory cytokines, proteases, reactive oxygen species (ROS) and 
senescent cells, the inability of dermal and/or epidermal cells to respond to reparative stimuli, and the presence of polymicrobial and persistent infections [1]. These biochemical alterations and unfavorable conditions, like moisture imbalance and alkaline $\mathrm{pH}$ ranging between 7.15-8.9, contribute to the prosperity of proteases and the reduction of oxygen dissociation from the hemoglobin molecules, which lead to a persistent inflammatory state, the hallmark of chronic nonhealing wounds [2]. Dressings play an important role in CW treatments. They allow the direct delivery of biomolecules to the affected site, protect the wound bed from physical and mechanical stress, guarantee a moisture environment and a provisional matrix for cell migration, deposition and neovascularization, while allowing appropriate levels of wound oxygenation. The architecture and the topography of the wound dressing play as well a significant role in wound healing due to its great influence on the parameters mentioned above. It is for being able to produce a 3D structure, with superior advantages to conventional dressings, such as high oxygen permeability, high surface to volume ratio, tunable pore size and structural similarity to the extracellular matrix (ECM) either from synthetic and either natural polymers, that electrospinning technique has been so prominent and attracted so much attention [3]. Cellulose acetate (CA), an ecofriendly polymer, is under great consideration in the biomedical industry due to its biocompatibility, biodegradability, high affinity to other polymers and biomolecules, good hydrolytic stability, relative low cost, excellent chemical resistance and mechanical performance, and ability to mimic the ECM to promote cell attachment, growth, and advanced formation of targeted tissues (e.g., bones and skin) [4,5]. Another polymer that continues to be frequently used in advanced biomedical applications is that poly(vinyl alcohol) (PVA), which is considered as one of the oldest synthetic polymers in existence (synthetized in 1924). PVA is a Food and Drug Administration (FDA)-approved polymer with excellent biocompatibility (non-toxic), biodegradability, hydrophilicity, transparency, good film forming abilities, thermostability, and chemical resistance. Despite the many advantages, its restricted strength and instant solubilization in aqueous environments has limited its application and has turned the crosslinking process into an indispensable step in dressing production [3,5]. Crosslinking implies the formation of chemical bonds between different molecular chains to generate strong, stable and water insoluble $3 \mathrm{D}$ networks. For PVA, the crosslinking process can be accomplished via chemical or physical reactions, being the dialdehydes, diisocyanates, dicarboxylic, tricarboxylic and boric acids the most frequent chemical agents applied [6]. Even though there are many options, glutaraldehyde (GA) is by far the most common crosslinker used in PVA processing due to its efficiency, ease of access and processing, and low cost. Further, in comparison with other crosslinking agents GA is also less cytotoxic (pivotal requirement). In fact, there are evidences that in its vapor form its inherent cytotoxic effect can be reduced or even neglected $[7,8]$. This manuscript aims to clarify the process of crosslinking with GA on mats made of PVA and CA. It also analyses the methodologies employed to prevent the accumulation of excess GA on electrospun meshes without compromising their functions.

\section{Methods: Crosslinking Process}

Electrospun PVA fibers may dissolve instantly upon contact with aqueous media, which can limit its use in many biomedical applications. To overcome this drawback, several studies have investigated the grafting of hydrophobic groups or crosslinking approachs to reach structures with poorer solubility and improved mechanical properties [8]. The crosslinking processes that have been described in the literature involve physical methods, such as heat and radiation or chemical procedures, as the immersion of membranes in organic acids, addition of a crosslinker/catalyst (usually a strong acid) to electrospinning solutions and exposure of electrospun membranes to reagent vapor [9]. Glutaraldehyde (GA), glyoxal, and boric acid have been some of chemical agents most used to PVA crosslink, but the GA due to its high efficiency in forming networks via the formation of covalent bonds, namely acetal bridges between the hydroxyl groups in PVA and CA and the difunctional aldehyde molecule of GA has taken on a relevant role in the reticulation of PVA [9]. However, GA has demonstrated some cytotoxicity to physiological tissues and therefore one of its approach that has been investigated is its vapor phase exposure to nanofibrous mats because it 
has less or no cytotoxic effect [10]. Moreover, post-treatment with other methods of detoxification are required in some cases to prevent the harmful effects of residual GA (unreacted or that has established intramolecular bonds) [8]. In the following section, it is described in which way we were able to attain an optimal balance of GA, in order to prevent excess in nanostructured mats and, later, problems of cytotoxicity, oxygen and humidity imbalance.

\section{Results and Discussion: Elimination of Excess GA}

To achieve well-reticulated meshes without excess of GA within their structure, various processes have been applied such as drying processes (using an oven or vacuum) [10,11], washes with glycine aqueous solution $[7,12,13]$, washes with distilled water or sodium bisulfite (SBS) [14], and storage conditions until the mats subsequent use [15]. In this work, the optimization processes were started with the 80/20 PVA/CA ratio, since a smaller number of hydroxyl groups were available to react with the aldehydes from GA. In a first approach, the vapor phase crosslinking was accomplished using $15 \mathrm{~mL}$ of GA $(2.56 \mathrm{M})$ and exposing the vacuum-sealed mats to RT or heat at 60 ${ }^{\circ} \mathrm{C}$ for 2, 4, 6, 7 and $8 \mathrm{~h}$. Samples processed at RT were unsuccessful. The optimal exposure time at $60{ }^{\circ} \mathrm{C}$ was established at $7 \mathrm{~h}$, since with smaller periods of time, the meshes were not efficiently reticulated, degrading upon contact with water, and after $8 \mathrm{~h}$ of treatment the mats gained a yellowish coloration. However, after crosslinking, the excess of GA was very visible, increasing considerably the average fiber diameter from 194 to $343 \mathrm{~nm}$ (on the ratio 80/20 PVA/CA). The meshes were submitted then, to various processes in attempt to remove GA excess: evaporation at $60^{\circ} \mathrm{C}$ and $45^{\circ} \mathrm{C}$ for $24 \mathrm{~h}$; and to three different washing procedures: (1) sonication at RT, (2) orbital shaking (100 rpm) at $37^{\circ} \mathrm{C}$, and washing in a $0.26 \mathrm{M}$ glycine solution with gentle orbital shaking (100 rpm) at RT for $30 \mathrm{~min}$. In the evaporation process at $60^{\circ} \mathrm{C}$, fibers became yellowish and rigid and at $45^{\circ} \mathrm{C}$ the mats retained their original color; but was not enough to eliminate efficiently the GA from the nanofibers. All washing procedures damaged the architecture and morphology of the membranes. To retain the original structure of the meshes, the reduction of the GA volume to $6 \mathrm{~mL}$ using a crosslinking process at 60 ${ }^{\circ} \mathrm{C}$ for $7 \mathrm{~h}$ was required. This was seen to maintain the morphology of the uncrosslinked meshes without any excess of GA (Figure 1) and to guarantee its stability in aqueous media. As extra content of GA was not detected on the fibers, drying and washing procedures were not employed. Still, possible GA residues could be eliminated during storage in a controlled environment $(41 \% \mathrm{RH}$ and $\left.19^{\circ} \mathrm{C}\right)$.

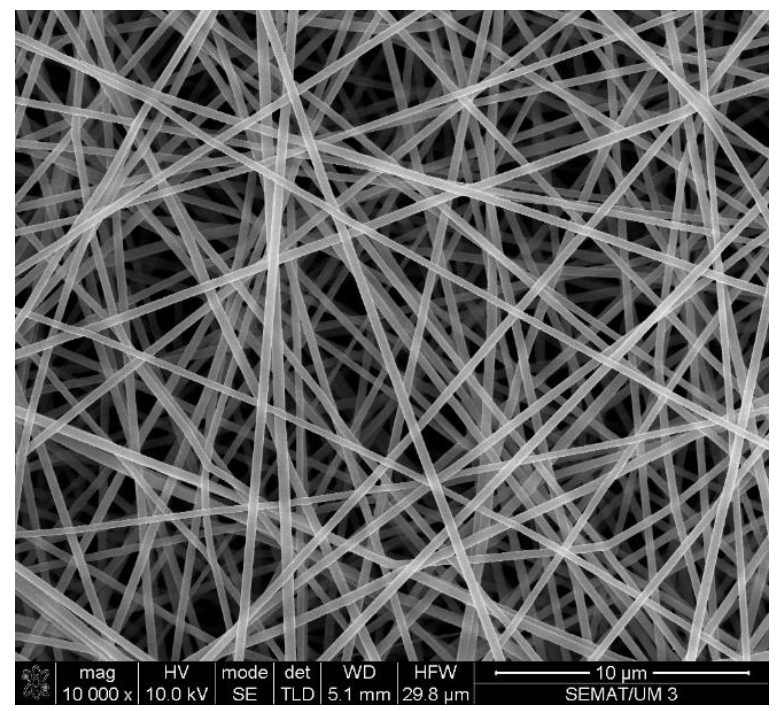

(a)

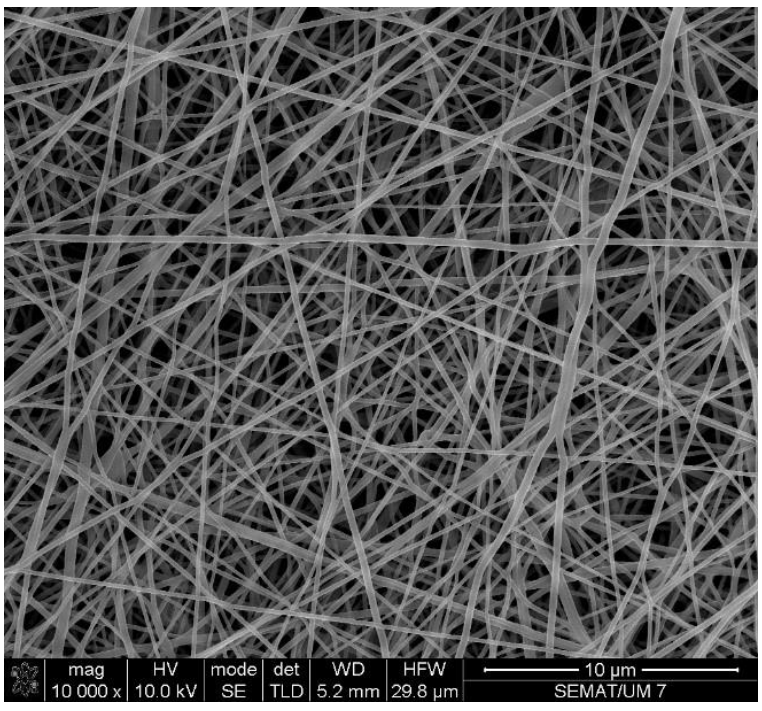

(b)

Figure 1. FEG-SEM micrograph of an 80/20 PVA/CA mat (10 000X magnification), (a) before and (b) after crosslinking using $6 \mathrm{~mL}$ of GA at $2.56 \mathrm{M}$. 


\section{Conclusions}

The objective of this work was to clarify about of various types and mechanisms of crosslinking that can be applied to PVA-based electrospun meshes, namely with GA vapor. Additionally, steps towards the complete elimination of GA retained within the mats were also explored. Optimal crosslinking processing conditions for PVA/CA blends were reached using the smallest amount of GA, namely $6 \mathrm{~mL}$, applied at $60^{\circ} \mathrm{C}$ for $7 \mathrm{~h}$. This volume, temperature and time were sufficient to establish acetal bonds between the hydroxyl groups of PVA and CA and the aldehyde groups of GA. Using these conditions, a stable and resilient fibrous network was attained capable of sustaining prolonged immersion in aqueous media. Further, the architecture of the meshes was not impaired due to the excess of GA, allowing the uniform porosity and the fibers morphology to prevail. These requirements are extremely important for applications in wound healing. Therefore, in addition to the several options described in the literature for removing excess GA, the amount of GA applied seems to be the simplest and most effective way to attain an effective crosslinking without harming the structure or turning the surface cytotoxic (due to excess GA).

Author Contributions: Conceptualization, M.A.T.; writing original draft, M.A.T.; revision and editing, J.C.A., H.P.F; supervision, H.P.F.; funding acquisition, M.T.P.A., H.P.F. All authors have read and agreed to the published version of the manuscript.

Funding: This research was funded by FCT, grant numbers PTDC/CTM-TEX/28074/2017 (POCI-01-0145-FEDER028074), and UID/CTM/00264/2020 and the scholarship SFRH/BD/148930/2019.

Acknowledgments: Authors acknowledge the Portuguese Foundation for Science and Technology (FCT), FEDER funds by means of Portugal 2020 Competitive Factors Operational Program (POCI) and the Portuguese Government (OE) for funding the project PEPTEX with reference PTDC/CTM-TEX/28074/2017 (POCI-01-0145FEDER-028074). Authors also acknowledge project UID/CTM/00264/2020 of Centre for Textile Science and Technology (2C2T), funded by national funds through FCT/MCTES. M.A.T. acknowledges FCT for the PhD grant with reference SFRH/BD/148930/2019. SEM studies were performed at the Materials Characterization Services of the University of Minho (SEMAT/UM).

Conflicts of Interest: The authors declare no conflict of interest.

\section{References}

1. Frykberg, R.G.; Banks, J. Challenges in the Treatment of Chronic Wounds. Adv. Wound Care 2015, 4, 560-582, doi:10.1089/wound.2015.0635.

2. Kim, H.S.; Sun, X.; Lee, J.H.; Kim, H.W.; Fu, X.; Leong, K.W. Advanced drug delivery systems and artificial skin grafts for skin wound healing. Adv. Drug Deliv. Rev. 2019, 146, 209-239, doi:10.1016/j.addr.2018.12.014.

3. Teixeira, M.A.; Amorim, M.T.P.; Felgueiras, H.P. Poly (Vinyl Alcohol) -Based Nanofibrous Electrospun Scaffolds for Tissue Engineering Applications. 2020, 12, 7, doi:10.3390/polym12010007.

4. Teixeira, M.A..; Amorim, M.T.P..; Felgueiras, H.P. Cellulose Acetate in Wound Dressings Formulations: Potentialities and Electrospinning Capability. In Proceedings of the XV Mediterranean Conference on Medical and Biological Engineering and Computing-MEDICON 2019; Springer International Publishing: Berlin/Heidelberg, Germany, 2020; Volume 76, pp. 1515-1525.

5. Felgueiras, H.P.; Teixeira, M.A.; Tavares, T.D.; Amorim, M.T.P. New method to produce poly(vinyl alcohol)/cellulose acetate films with improved antibacterial action. Mater. Today Proc. 2020, 10-13, doi:10.1016/j.matpr.2019.12.100.

6. Shaikh, R.P.; Kumar, P.; Choonara, Y.E.; Du Toit, L.C.; Pillay, V. Crosslinked electrospun PVA nanofibrous membranes: Elucidation of their physicochemical, physicomechanical and molecular disposition. Biofabrication 2012, 4, doi:10.1088/1758-5082/4/2/025002.

7. Akhshabi, S.; Biazar, E.; Singh, V.; Keshel, S.H. The effect of glutaraldehyde cross-linker on structural and biocompatibility properties of collagen-chondroitin sulfate electrospun mat. Mater. Technol. 2018, 7857, 19, doi:10.1080/10667857.2017.1410998.

8. Destaye, A.G.; Lin, C.; Lee, C. Glutaraldehyde Vapor Cross-linked Nanofibrous PVA Mat with in Situ Formed Silver Nanoparticles. ACS Appl. Mater. Interfaces 2013, 5, 4745-4752, https://doi.org/10.1021/am401730x . 
9. Truong, Y.B.; Choi, J.; Mardel, J.; Gao, Y.; Maisch, S.; Musameh, M.; Kyratzis, I.L. Functional Cross- Linked Electrospun Polyvinyl Alcohol Membranes and Their Potential Applications. Macromol. Mater. Eng. 2017, 302, 1-9, doi:10.1002/mame.201700024.

10. Wang, W.; Jin, X.; Zhu, Y.; Zhu, C.; Yang, J.; Wang, H.; Lin, T. Effect of vapor-phase glutaraldehyde crosslinking on electrospun starch fibers. Carbohydr. Polym. 2016, 140, 356-361, doi:10.1016/j.carbpol.2015.12.061.

11. Wang, P.H.; Huang, B.S.; Horng, H.C.; Yeh, C.C.; Chen, Y.J. Wound healing. J. Chinese Med. Assoc. 2018, 81, 94-101, doi:10.1016/j.jcma.2017.11.002.

12. Alhosseini, S.N.; Moztarzadeh, F.; Mozafari, M.; Asgari, S.; Dodel, M.; Samadikuchaksaraei, A.; Kargozar, S.; Jalali, N. Synthesis and characterization of electrospun polyvinyl alcohol nanofibrous scaffolds modified by blending with chitosan for neural tissue engineering. Int. J. Nanomedicine 2012, 7, 25-34, doi:10.2147/IJN.S25376.

13. Mohammadzadehmoghadam, S.; Dong, Y. Fabrication and Characterization of Electrospun Silk Fibroin/Gelatin Scaffolds Crosslinked With Glutaraldehyde Vapor. Front. Mater. 2019, 6, 1-12, doi:10.3389/fmats.2019.00091.

14. Plan, S.C. Spills, Deactivation, and Disposal of Glutaraldehyde. Dow Chem. Co. 2017. pp. 4-6. Available online: $\quad$ https://www.metrex.com/sites/default/files/content/education-file/education-fileupload/glutaraldehydespills-dowbiosharedatasheets.pdf (accessed on).

15. Rojas-Mercado, A.S.; Moreno-Cortez, I.E.; Lucio-Porto, R.; Pavón, L.L. Encapsulation and immobilization of ficin extract in electrospun polymeric nanofibers. Int. J. Biol. Macromol. 2018, 118, 2287-2295, doi:10.1016/j.ijbiomac.2018.07.113.

Publisher's Note: MDPI stays neutral with regard to jurisdictional claims in published maps and institutional affiliations.

(C) 2020 by the authors. Submitted for possible open access publication under the terms and conditions of the Creative Commons Attribution (CC BY) license (http://creativecommons.org/licenses/by/4.0/). 